\title{
Simultaneous Observation Data of GB-SAR/PiSAR to Detect Flooding in an Urban Area
}

\author{
Manabu Watanabe, ${ }^{1}$ Masayoshi Matsumoto, ${ }^{1}$ Masanobu Shimada, ${ }^{2}$ Tomohito Asaka, ${ }^{3}$ \\ Hajime Nishikawa, ${ }^{3}$ and Motoyuki Sato ${ }^{1}$ \\ ${ }^{1}$ CNEAS, Tohoku University, Sendai 980-8576, Japan \\ ${ }^{2}$ EORC/JAXA, Tsukuba 305-8505, Japan \\ ${ }^{3}$ Department of Civil Engineering, Nihon University, Narashino 275-8575, Japan
}

Correspondence should be addressed to Manabu Watanabe, mwatana@cneas.tohoku.ac.jp

Received 1 June 2009; Revised 29 August 2009; Accepted 4 November 2009

Academic Editor: Carlos Lopez-Martinez

Copyright (C) 2010 Manabu Watanabe et al. This is an open access article distributed under the Creative Commons Attribution License, which permits unrestricted use, distribution, and reproduction in any medium, provided the original work is properly cited.

We analyzed simultaneous observation data with ground-based synthetic aperture radar (GB-SAR) and airborne SAR (PiSAR) over a flood test site at which a simple house was constructed in a field. The PiSAR $\sigma^{\circ}$ under flood condition was 0.9 to $3.4 \mathrm{~dB}$ higher than that under nonflood condition. GB-SAR gives high spatial resolution as we could identify a single scattering component and a double bounce component from the house. GB-SAR showed that the $\sigma^{\circ}$ difference between the flooding and nonflooding conditions came from the double bounce scattering. We also confirm that the entropy is a sensitive parameter in the eigenvalue decomposition parameters, if the scattering process is dominated by the double bounce scattering. We conclude that $\sigma^{\circ}$ and entropy are a good parameter to be used to detect flooding, not only in agricultural and forest regions, but also in urban areas. We also conclude that GB-SAR is a powerful tool to supplement satellite and airborne observation, which has a relatively low spatial resolution.

\section{Introduction}

Synthetic aperture radar (SAR) can provide important information for calculating the extent of flooding, since it is not limited by weather or time of day. The extent is calculated from SAR data taken before and after flooding. But it is sometimes difficult to distinguish the flooded area just from SAR backscattering, because waves caused by wind cause a bright reflection and thus misclassification. The simultaneous use of optical sensors or GIS data has been proposed for reducing misclassification [1-3].

ALOS/PALSAR, launched in 2006, could be useful for flood monitoring. Several emergency observations of flood disaster sites have been done, including in Myanmar (Burma) on 2008 May 6, and in New Brunswick, Canada, on 2008 May 5. Although several floods in open areas have been examined [4], little attention has been given to flooding in urban areas. This is because it is difficult to get verification data in urban areas, and there have been few observations by satellite or airborne SAR during such floods.
On the other hand, several ground-based (GB) SAR systems have been developed as tools for monitoring agriculture and large built structures and for terrain mapping. Pieraccini et al. [5] proposed the GB interferometric SAR technique for terrain mapping. Tarchi et al. [6] described an innovative application of radar interferometric techniques aimed at monitoring structural deformation of buildings in cultural heritage surveys. GB-SAR might supplement satellite/airborne observation, which has relatively low spatial resolution and limited chance of observation. But few simultaneous observations using satellite/airborne SAR and GB-SAR have been carried out.

Here, we analyze the results of full polarimetric data taken with GB-SAR and airborne SAR in an artificially made urban flood area. We discuss the scattering process as measured in L-band full polarimetry mode, introduce the PiSAR and GB-SAR system, and describe a preliminary experiment and observation of the area with PiSAR and GBSAR. 
TABle 1: Characteristics of PiSAR and GB-SAR.

\begin{tabular}{lcc}
\hline & PiSAR (L-band) & GB-SAR \\
\hline Frequency & $1.27 \mathrm{GHz}$ & $0.5-6.3 \mathrm{GHz}$ (antenna) \\
& & $0.05-20.05 \mathrm{GHz}$ (VNA) \\
Band width & $50 \mathrm{MHz}$ & Variable \\
Resolution & $2.5 \mathrm{~m}$ & $\sim 10 \mathrm{~cm}$ \\
Polarization & Full polarimetry & \\
\hline
\end{tabular}

VNA: vector network analyzer.

TABLE 2: $\sigma^{\circ}$ derived from double scattering of asphalt wall and metal sheet with GB-SAR.

\begin{tabular}{lccc}
\hline & $\sigma^{\circ}{ }_{\mathrm{HH}}$ & $\sigma^{\circ}{ }_{\mathrm{HV}}$ & $\sigma^{\circ} \mathrm{VV}$ \\
\hline Asphalt wall (dB) & -36.0 & -47.3 & -38.9 \\
Metal sheet wall (dB) & -33.1 & -43.1 & -33.9 \\
Diff. (dB) & 2.9 & 4.2 & 5.0 \\
\hline
\end{tabular}

\section{PISAR and GB-SAR}

Characteristics of PiSAR and GB-SAR are summarized in Table 1. PiSAR is a Japanese airborne SAR that carries an L-band SAR from the Japan Aerospace Exploration Agency (JAXA) and an X-band SAR from the National Institute of Information and Communication Technology (NICT). We used the L-band SAR data. The GB-SAR system consists of a vector network analyzer (VNA), a double polarized diagonal horn antenna with the size of $47 \mathrm{~cm}$, an antenna positioner unit, and a PC-based control unit. The VNA, operated in stepped-frequency continuous-wave mode, generates the transmitting signal and detects signals scattered in both amplitude and phase.

We tested the polarimetric radiometric performance of the GB-SAR system by using the reflections from a metal plane. The channel imbalance, $\sigma^{\circ}{ }_{\mathrm{VV}} / \sigma^{\circ} \mathrm{HH}$, was less than $1 \mathrm{~dB}$; crosstalk, $\sigma^{\circ} \mathrm{HV} / \sigma^{\circ} \mathrm{HH}$, was about $-45 \mathrm{~dB}$. Other characteristics are presented by Zhou [7].

To test the suitability of GB-SAR to detect flooding, we examined double bounce scattering from a concrete wall and an asphalt ground surface (Figure 1). Instead of water, we laid a $1-\mathrm{m} \times 1-\mathrm{m}$ metal sheet on the ground, and examined the change in reflection power from the double bounce scattering. The data were collected in the frequency range of $86 \mathrm{MHz}$ to $5.68 \mathrm{GHz}$. After applying a bandpass filter of 0.8 to $1.8 \mathrm{GHz}$, which corresponds to the L-band, we used inverse Fourier transformation to create time domain data [8]. We used a migration method of diffraction stacking to reconstruct the image $[9,10]$. The raw backscattering coefficient $\left(\sigma^{\circ}\right)$, which consist of products of the backscattering coefficient $\left(\sigma^{\circ}\right)$ from the target, antenna gain, and effective area are summarized in Table 2. Bright reflections were observed in the like polarization, as expected, but some cross-polarization reflection also appeared, owing to dihedral reflection at some incident azimuth angles caused by the wide antenna pattern of $\sim 60^{\circ}$.
By using simple double bounce reflection model and Fresnel reflection, we estimate the difference of $\sigma^{\circ}$ acquired from asphalt-wall and metal-wall reflection. Dielectric constant of the wall is assumed to be 10 , and reflectivity of the metal sheet is 1 . The estimated differences are $8.8 \mathrm{~dB}$ and $11.4 \mathrm{~dB}$ in $\mathrm{HH}$ and $\mathrm{VV}$ polarization and are about $6 \mathrm{~dB}$ larger than those acquired by GB-SAR. These may be due to a contamination of other scattering component for the GBSAR experiment, such as edge reflection of the metal sheet. On the other hand, the $\sigma^{\circ} \mathrm{VV}$ became lower than the $\sigma^{\circ} \mathrm{HH}$ in both cases. This is explained by a lower reflectivity of VV polarization by an effect of the Brewster angle. We conclude that our GB-SAR system is capable of detecting flooding by a way of double scattering from ground and walls. We expect some difference in $\sigma^{\circ}$ values between flooding and nonflooding situations in urban areas.

\section{Observations}

We built a simple house in a field of Nihon University, Chiba, Japan. The house measured $5 \mathrm{~m} \times 5 \mathrm{~m} \times 5 \mathrm{~m}$ and was covered with thin wooden board $3 \mathrm{~mm}$ thick. The roof was made of thin zinc plate. Water was poured around the house to mimic a flood. Several PiSAR observations were performed over 3 days before and after the flooding with L-band SAR in full polarimetry mode.

The first PiSAR observations were done on 2007 November 27, when the soil moisture content around the house was $\sim 20 \%$. The next observations were done on November 29 , when the area surrounding the house was covered with water (moisture content 100\%). The third observations were done on November 30, after the water was drained but the soil remained wet $(47.4 \% \pm 4.0 \%)$. Several observations were collected in different flight directions at off-nadir angles of $30^{\circ}$ and $45^{\circ}$. We used data with the same flight direction at an off-nadir angle of $45^{\circ}$. All three PiSAR images show bright reflections from the house and two of them are shown in Figure 2, which are not geometrically corrected. Geometric differences between the November 27 image and the other two images may be due to a slightly different azimuth angle, so we used only the data taken on November 29 and 30 . We also collected simultaneous GB-SAR data on November 29 and 30 to see detailed radar reflections from the house. The GB-SAR antenna was pointed at the base of the house at an off-nadir angle of $80^{\circ}$, since we could not deploy the GB-SAR in the water (Figure 3 ). The acquired frequency domain data were processed as described above, and radar images were reconstructed from the original data.

\section{Results and Discussion}

Full polarimetry images derived from GB-SAR are shown in Figure 4. The horizontal axis is a range distance ranging from $9 \mathrm{~m}$ to $16 \mathrm{~m}$, and the vertical axis is an azimuth distance ranging from $1.5 \mathrm{~m}$ to $7.5 \mathrm{~m}$. Since the wooden board is thin, we can see the reflection from the metal bars behind it. The horizontal bars are well detected in the $\mathrm{HH}$ image, and the vertical bars are partially detected in the VV image. 


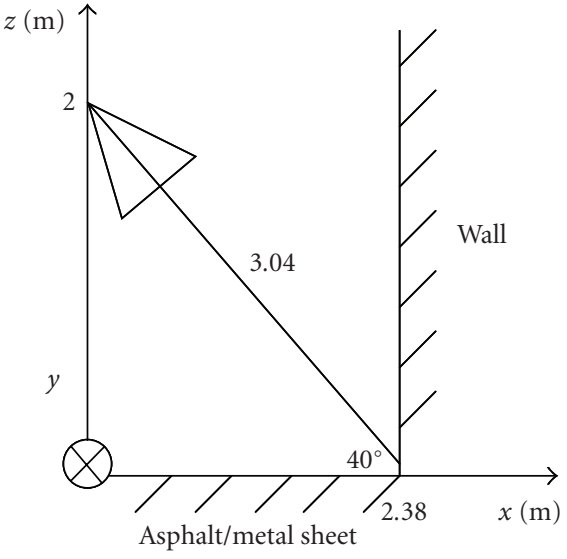

(a)

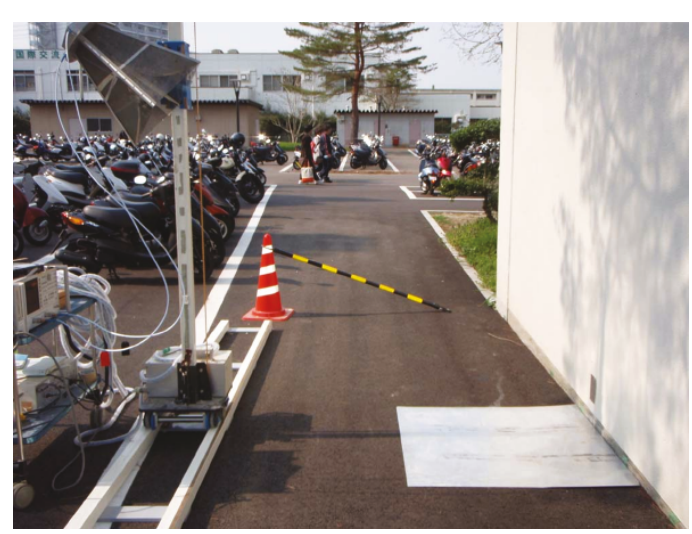

(b)

FIGURE 1: Configuration and photo of the preliminary experiment to test double bounce scattering with GB-SAR.

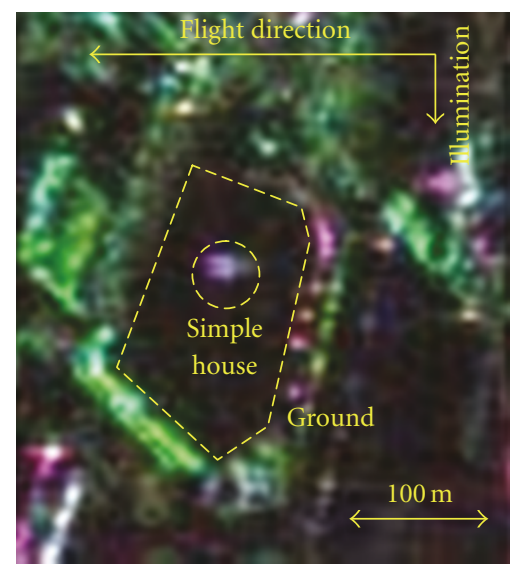

$11 / 29$ (flooded)

(a)

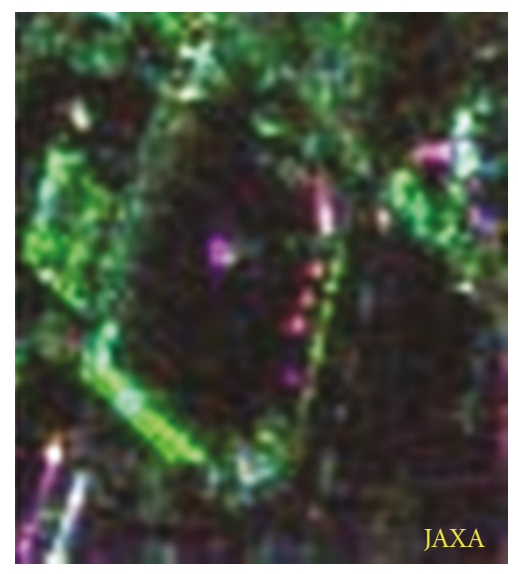

$11 / 30$ (wet soil)

(b)

FIgURe 2: PiSAR L-band images on 2007 November 27 (left), 29 (middle), and 30 (right). Red: HH; green: HV; blue: VV.

Values of $\sigma^{\circ}$, entropy (H), $\alpha$, and anisotropy (A) derived from the PiSAR data are summarized in Table 3. Since PiSAR does not have enough spatial resolution to separate the house structure, we used all signal from the house in the analysis. The $\sigma^{\circ}$ values of the $\mathrm{HH}$ and VV polarizations on November 29 are 0.9 , and $1.5 \mathrm{~dB}$ higher than those on November 30 . That of the HV polarization was $3.4 \mathrm{~dB}$ higher on November 29. These changes are simply explained by the higher reflectivity of the water surface on November 29.

When we plot $\alpha$ and $\mathrm{H}$ taken with PiSAR in an $\alpha-\mathrm{H}$ plane, which is proposed by Cloude and Pottier [11], the $\mathrm{H}$ and the $\alpha$ values taken in both flooding and nonflooding conditions, are categorized in $\mathrm{z} 9$ zone, which include GO and PO surface scattering, Bragg surface scattering, and specular scattering. This classification and observation configuration indicate that dominant scattering process comes from a single scattering from the roof of the simple house, while single scattering from the metal bars and double scattering from the metal bar and the surface are included in some degree. The $\mathrm{H}$ shows almost same value, and the $\alpha$ show 5 degree lower on November 30th. I will discuss these with the GB-SAR results later.

Parameters derived from GB-SAR are also summarized in Table 3. We calculated $\sigma^{\circ}, \mathrm{H}, \alpha$, and $\mathrm{A}$ in two regions in the GB-SAR image (Figure 4). Reg. 2 is located at the range distance of $10 \mathrm{~m}$ and comes from the double bounce scattering (Figure 5). The $\sigma^{\circ}{ }_{\mathrm{HH}}$ shows a 9.3-dB difference between flooding and nonflooding conditions. This increase can be explained by specular reflection on the water surface during the flood. Reg. 1 signal is located at the range distance of $9.8 \mathrm{~m}$ and comes from the single bounce scattering from the nearest metal bar (Figure 5). We found no difference in the $\sigma^{\circ} \mathrm{HH}$ taken before and after the flooding because the flooding does not affect this scattering process. Since the metal bar of Reg. 1 is horizontal, few $\sigma^{\circ} \mathrm{Vv}$ values were recorded. A 1.4-dB difference in cross-polarization at Reg. 1 


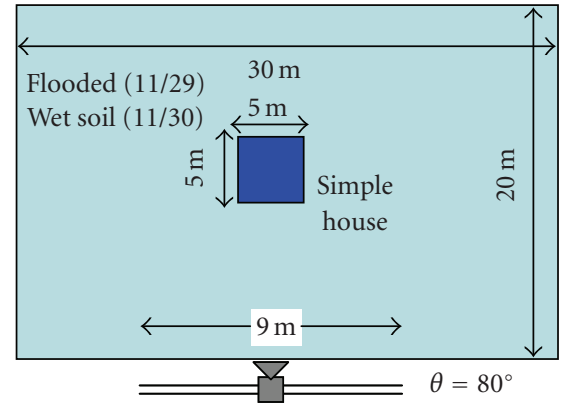

(a)

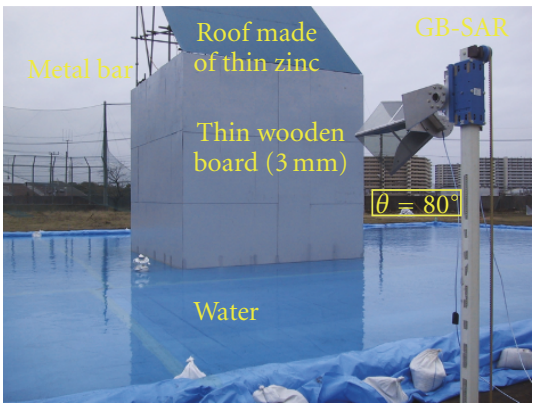

(b)

FIgURE 3: (a) Configuration of the field experiment. (b) Photo of the simple house and GB-SAR, which was deployed outside the water.

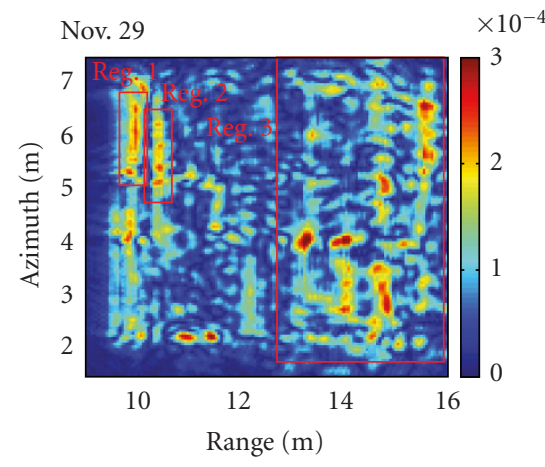

(a)

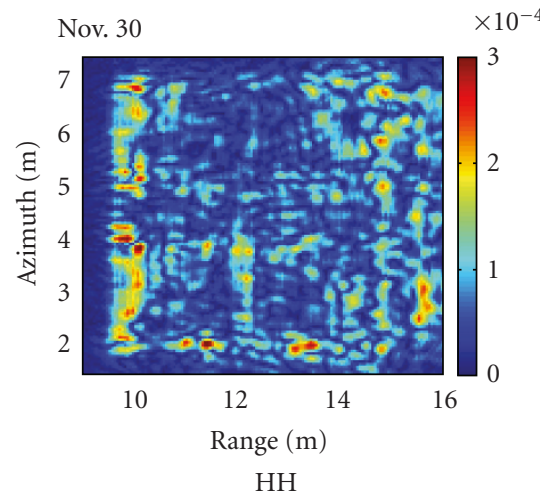

(d)

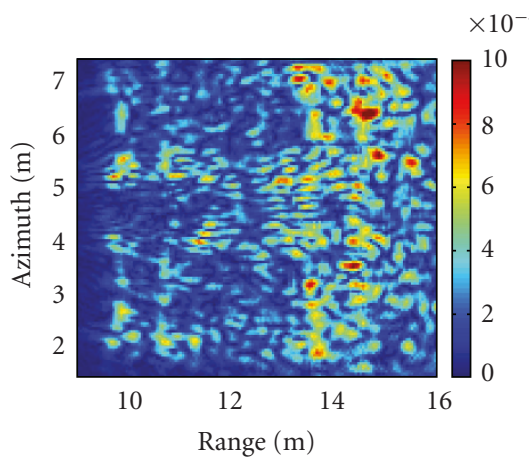

(b)

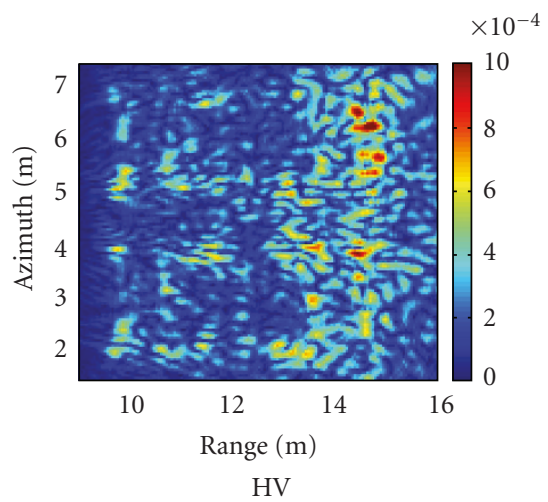

(e)

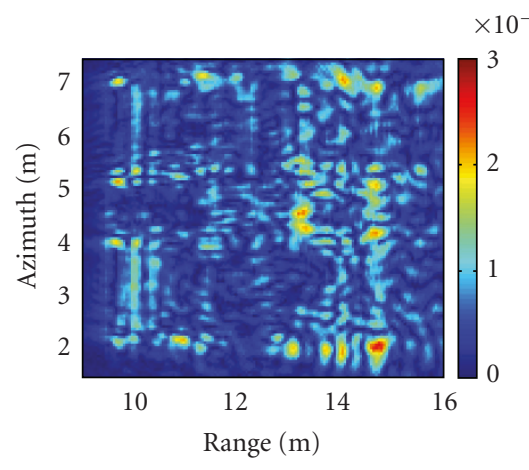

(c)

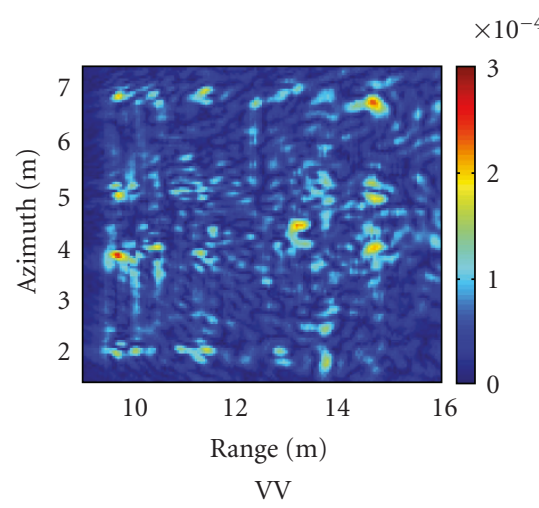

(f)

FIGURE 4: Reflections from simple house taken by GB SAR in HH, HV, and VV polarization.

might be due to the different incident azimuth angle between November 29 and 30, or reflection from the horizontal wire with some width.

We again use the double scattering model and examine the difference of the $\sigma^{\circ} \mathrm{HH}$ for the flooding/nonflooding condition. The dielectric constant of the soil is estimated as 32 from soil moisture value [12] and that of the water is 80 .

The GB-SAR Case. The $\sigma^{\circ} \mathrm{HH}$ difference for the model is $0.4 \mathrm{~dB}$, while that acquired with GB-SAR measurement is $9.0 \mathrm{~dB}$. One of the possible explanations for this discrepancy is a roughness of the soil, which reduce the reflectivity of the soil surface.
PiSAR Case. The $\sigma^{\circ}{ }_{\mathrm{HH}}$ difference is $1.6 \mathrm{~dB}$, while that acquired with PiSAR measurement is $0.9 \mathrm{~dB}$, and is smaller than the model value in this case. This discrepancy can be explained that the double scattering component is a part of the component for the PiSAR case, and other scattering process, such as single scattering, is a dominant scattering process.

The GB-SAR radar signals in Reg. 3 derive from various kinds of scattering processes in the house. Because the structure of the metal bars in the house is complex, we could not identify the scattering source. But the average $\sigma^{\circ}$ values increased after the flooding. 
TABle 3: Parameters derived from GB-SAR and PiSAR.

\begin{tabular}{|c|c|c|c|c|c|c|c|}
\hline & \multicolumn{4}{|c|}{ GB-SAR } & \multicolumn{3}{|c|}{ PiSAR } \\
\hline & & November 29 & November 30 & Diff. & November 29 & November 30 & Diff. \\
\hline \multirow{2}{*}{$\sigma_{\mathrm{HH}}^{\circ}(\mathrm{dB})$} & Reg. 1 & -77.4 & -77.6 & 0.2 & \multirow{2}{*}{30.4} & \multirow{2}{*}{29.5} & \multirow{2}{*}{0.9} \\
\hline & Reg. 2 & -76.0 & -85.3 & 9.3 & & & \\
\hline \multirow{2}{*}{$\sigma^{\circ}{ }_{\mathrm{HV}}(\mathrm{dB})$} & Reg. 1 & -91.7 & -93.1 & 1.4 & \multirow{2}{*}{18.6} & \multirow{2}{*}{15.1} & \multirow{2}{*}{3.4} \\
\hline & Reg. 2 & -96.2 & -96.7 & 0.5 & & & \\
\hline \multirow{2}{*}{$\sigma^{\circ}{ }_{\mathrm{VV}}(\mathrm{dB})$} & Reg. 1 & -88.3 & -88.1 & -0.2 & \multirow{2}{*}{31.6} & \multirow{2}{*}{30.1} & \multirow{2}{*}{1.5} \\
\hline & Reg. 2 & -86.2 & -86.3 & 0.1 & & & \\
\hline \multirow{2}{*}{ Entropy $(\mathrm{H})$} & Reg. 1 & 0.29 & 0.36 & -0.07 & \multirow{2}{*}{0.38} & \multirow{2}{*}{0.37} & \multirow{2}{*}{0.01} \\
\hline & Reg. 2 & 0.41 & 0.66 & -0.25 & & & \\
\hline \multirow{2}{*}{$\alpha(0)$} & Reg. 1 & 40.1 & 52.5 & -12.4 & \multirow{2}{*}{22.9} & \multirow{2}{*}{17.8} & \multirow{2}{*}{5.0} \\
\hline & Reg. 2 & 51.3 & 47.3 & 4.0 & & & \\
\hline \multirow[t]{2}{*}{ Anisotropy (A) } & Reg. 1 & 0.56 & 0.72 & -0.16 & \multirow[t]{2}{*}{0.71} & \multirow[t]{2}{*}{0.74} & \multirow[t]{2}{*}{0.03} \\
\hline & Reg. 2 & 0.45 & 0.61 & -0.15 & & & \\
\hline
\end{tabular}

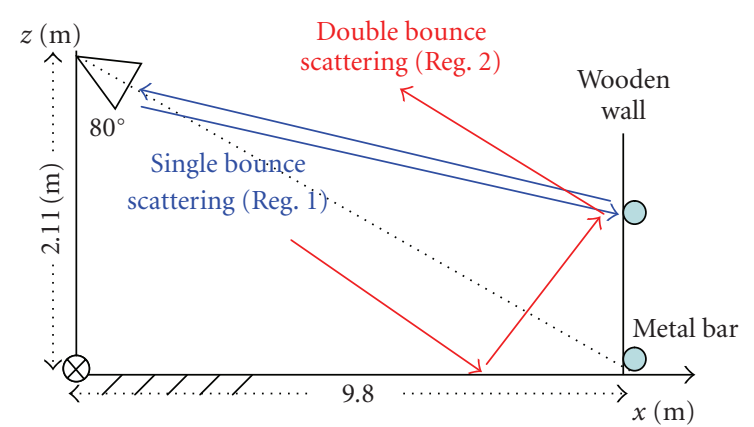

Figure 5: Single and double bounce scattering from the ground surface and metal bars.

The $\alpha$ values derived from Reg. 1 and Reg. 2 are $40.1^{\circ}$ and $52.5^{\circ}$, and these are well explained by the characteristics of the $45^{\circ} \alpha$ parameter, which includes the reflection from the isolated dipole.

The reflection process of Reg. 2 is changing from $\mathrm{z} 7$ for the flooding to $\mathrm{z} 5$ for the nonflooding in the $\alpha-\mathrm{H}$ plane. Significant change is seen for $\mathrm{H}$, which is changing from 0.41 to 0.66 . Because the nonflooding double bounce reflection includes the reflection of the soil surface, it might increase the $\mathrm{H}$ value. The reflection process of Reg. 1 is changing from $\mathrm{z} 9$ to $\mathrm{z} 7$. But the $\mathrm{H}$ does not show large change between flooding $(0.29)$ and nonflooding $(0.36)$ conditions and shows approximately same value with the one derived from the PiSAR data. These results also support the idea that Reg. 1 and dominant component of the PiSAR data are the single scattering from the house. But we have to note that many of the house's roof is not made of metal like in this experiment. If there is no strong reflection from the roof, entropy change derived with SAR data may be useful parameters to detect the flooding, as shown in the GB-SAR case. And $\alpha-\mathrm{H}$ classification scheme is a good indicator of the reflection process so that we can judge whether the entropy can be applied or not for flood detection.
On the other hand, the change of the $\alpha$ between the flooding and nonflooding conditions shows complicated behavior. The $\alpha$ value for the flooding condition is $4-5^{\circ}$ higher than that for the nonflooding condition in the PiSAR data and in the Reg. 2 of the GB-SAR data. On the other hand, Reg. 1 shows $12.4^{\circ}$ which is a lower value. These may be due to a slight difference in the repeat-pass observation geometry.

\section{Conclusion}

We performed a simultaneous GB-SAR/PiSAR experiment over a flood test site with a simple house. Several PiSAR observations were done over 3 days with different soil moisture conditions. Additional GB-SAR observations were done on 2 of the 3 days at high spatial resolution. The PiSAR $\sigma^{\circ}$ under flood condition was 0.9 to $3.4 \mathrm{~dB}$ higher than that under nonflood condition. The higher-resolution GB-SAR data revealed a single scattering component from metal bars in the walls and a double bounce component from the ground surface and the metal bars. The GB-SAR data showed that the $\sigma^{\circ}$ difference arose from the double bounce scattering from the surface and metal bars. The $\alpha$ $\mathrm{H}$ classification suggests that dominant scattering process of the PiSAR data is the roof of the house. On the other hand, $\mathrm{H}$ and $\alpha$ values acquired with GB-SAR are well described by the single bounce from the dipole or the double scattering from the dipole and soil/water surface. Our analysis results show that the entropy is a sensitive parameter for the flood detection, if the scattering process is dominated by the double bounce scattering. And $\alpha-\mathrm{H}$ classification scheme is a well indicator of a reflection source, and whether we can use the entropy or not.

We conclude that $\sigma^{\circ}$ and entropy taken before and after a flood are useful for detecting flooded areas, not only in agricultural and forest regions, but also in urban areas. We also conclude that GB-SAR is a powerful tool to supplement satellite and airborne observations, which have relatively low spatial resolution. 


\section{Acknowledgments}

The authors would like to thank the Disaster Management Support Systems Office of JAXA for providing them with the opportunity to do the experiment. They are also grateful to Professor H. Nishikawa's laboratory members at Nihon University, who assisted with their field activities. The field experiment was funded by JAXA. The authors would also like to thank the PiSAR flight team at JAXA NICT for providing the data. This work was supported by a JSPS Grant-in-Aid for Scientific Research (S) 18106008.

\section{References}

[1] S. Takeuchi, T. Konishi, Y. Suga, and S. Kishi, "Comparative study for flood detection using JERS-1 SAR and Landsat TM data," in Proceedings of IEEE International Geoscience and Remote Sensing Symposium (IGARSS '99), vol. 2, pp. 873-875, Hamburg, Germany, June-July 1999.

[2] Y. Sun, X. Li, H. Gong, W. Zhao, and Z. Gong, "A study on optical and SAR data fusion for extracting flooded area," in Proceedings of IEEE International Geoscience and Remote Sensing Symposium (IGARSS '07), pp. 3086-3089, Barcelona, Spain, June 2007.

[3] C. J. Yang, Y. M. Wei, S. Y. Wang, Z. X. Zhang, and S. F. Huang, "Extracting the flood extent from satellite SAR image with the support of topographic data," in Proceedings of the International Conferences on Info-Tech and Info-Net (ICII '01), vol. 1, pp. 87-92, Beijing, China, October-November 2001.

[4] M. Ohki, M. Shimada, O. Isoguchi, and N. Kawano, "ALOS/PALSAR observation of flood disasters in 2008: early results and evaluation," in Proceedings of the 45th Autumn Conference of the Remote Sensing Society of Japan, pp. 215-216, Ebetsu, Japan, December 2008.

[5] M. Pieraccini, G. Luzi, and C. Atzeni, "Terrain mapping by ground-based interferometric radar," IEEE Transactions on Geoscience and Remote Sensing, vol. 39, no. 10, pp. 2176-2181, 2001.

[6] D. Tarchi, H. Rudolf, M. Pieraccini, and C. Atzeni, "Remote monitoring of buildings using a ground-based SAR: application to cultural heritage survey," International Journal of Remote Sensing, vol. 21, no. 18, pp. 3545-3551, 2000.

[7] Z.-S. Zhou, Application of a ground-based SAR, Ph.D. dissertation, Tohoku University, Sendai, Japan, 2003.

[8] R. N. Bracewell, The Fourier Transform and Its Applications, McGraw Hill, New York, NY, USA, 2nd edition, 1986.

[9] Z.-S. Zhou and M. Sato, "Development and performance evaluation of a ground-based SAR system," IEIC Technical Report, vol. 102, no. 84, pp. 31-36, 2002.

[10] Ö. Yilmaz, Seismic Data Processing, Society of Exploration Geophysicists, Tulsa, Okla, USA, 1987.

[11] S. R. Cloude and E. Pottier, "An entropy based classification scheme for land applications of polarimetric SAR," IEEE Transactions on Geoscience and Remote Sensing, vol. 35, no. 1, pp. 68-78, 1997.

[12] G. C. Topp, J. L. Davis, and A. P. Annan, "Electromagnetic determination of soil water content: measurements in coaxial transmission lines," Water Resources Research, vol. 16, no. 3, pp. 574-582, 1980 